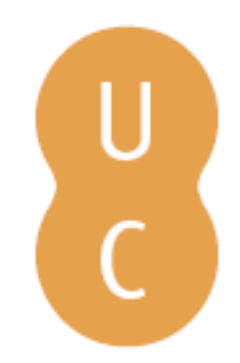

\title{
pommalina
}

\section{O acesso aberto e o futuro da investigação e comunicação científica}

\author{
Autor(es): $\quad$ Rodrigues, Eloy \\ Publicado por: Imprensa da Universidade de Coimbra \\ URL \\ persistente: \\ URI:http://hdl.handle.net/10316.2/36983 \\ DOI: \\ DOI:http://dx.doi.org/10.14195/978-989-26-1045-0_12
}

Accessed : $\quad$ 26-Apr-2023 12:18:01

A navegação consulta e descarregamento dos títulos inseridos nas Bibliotecas Digitais UC Digitalis, UC Pombalina e UC Impactum, pressupõem a aceitação plena e sem reservas dos Termos e Condições de Uso destas Bibliotecas Digitais, disponíveis em https://digitalis.uc.pt/pt-pt/termos.

Conforme exposto nos referidos Termos e Condições de Uso, o descarregamento de títulos de acesso restrito requer uma licença válida de autorização devendo o utilizador aceder ao(s) documento(s) a partir de um endereço de IP da instituição detentora da supramencionada licença.

Ao utilizador é apenas permitido o descarregamento para uso pessoal, pelo que o emprego do(s) título(s) descarregado(s) para outro fim, designadamente comercial, carece de autorização do respetivo autor ou editor da obra.

Na medida em que todas as obras da UC Digitalis se encontram protegidas pelo Código do Direito de Autor e Direitos Conexos e demais legislação aplicável, toda a cópia, parcial ou total, deste documento, nos casos em que é legalmente admitida, deverá conter ou fazer-se acompanhar por este aviso. 

Tendo como pano de fundo as Comemorações dos seus 500 anos, a Biblioteca Geral da Universidade de Coimbra organizou um Congresso Internacional subordinado ao tema "A Biblioteca da Universidade: permanência e metamorfoses", que teve lugar nos dias 16, 17 e 18 de janeiro de 2014, no auditório da Reitoria da Universidade de Coimbra.

O objetivo maior desta reunião científica foi o de refletir sobre o presente e o futuro das bibliotecas que servem públicos universitários. Numa outra vertente, procurou chamar-se a atenção para a importância de que a Biblioteca se reveste, tendo em vista o progresso do conhecimento técnico e científico. Por último, o Congresso pretendeu instituir-se como oportunidade de reflexão prospetiva e como lugar de encontro entre as sensibilidades de todos os que trabalham profissionalmente com livros e com outros suportes de natureza bibliográfica.

Nesse sentido, foram apresentadas Conferências, Mesas Redondas e sessões de Testemunhos em torno de temas como o valor das bibliotecas universitárias, a biblioteca universitária em contexto; as mudanças e os desafios; a biblioteca universitária e a sociedade da informação e conhecimento; o impacto do acesso aberto na comunidade científica, e as bibliotecas digitais. 


\title{
ELOY RODRIGUES
}

Universidade do Minho

University of Minbo

\section{O ACESSO ABERTO E O FUTURO DA INVESTIGAÇÃO E COMUNICAÇ ÃO C I E NTÍ́FICA}

\author{
OPEN ACCESS AND THE FUTURE OF RESEARCH \\ ANDSCIENTIFIC COMMUNICATION
}

\begin{abstract}
RESUMO: O acesso aberto aos resultados da investigação académica e científica conheceu um notável progresso na última década, desde que o conceito de open access foi definido e divulgado através da Declaração de Budapeste em 2002. Hoje parece cada vez mais provável que o acesso aberto será a forma predominante na comunicação científica no horizonte de uma década.

Mas estamos ainda a meio do período de transição e existe muita incerteza quanto ao caminho que irá ser trilhado nos próximos anos. A transição poderá ser comandada pelos interesses da investigação (com os investigadores e as organizações de investigação, como as universidades, a assumir maior responsabilidade e protagonismo na disseminação e publicação dos seus próprios resultados), ou poderá ser realizada sob a direção da indústria da publicação científica. Disso dependerá, por um lado, a configuração final do sistema de comunicação científica em acesso aberto e, por outro, o papel que poderá estar reservado às bibliotecas universitárias nesse sistema.

À luz da experiência dos últimos dez anos, e especialmente dos desenvolvimentos mais recentes nas políticas de acesso aberto dos organismos financiadores de ciência, nesta comunicação procuramos refletir sobre as ameaças e oportunidades, em particular para as bibliotecas universitárias, da transição para o acesso aberto.
\end{abstract}

ABSCTRACT: Open access to the results of academic and scientific research has made a remarkable progress since the concept was defined and published in the 2002 Budapest Declaration. Nowadays it seems increasingly likely that open access will be the prevailing form on scientific communication within a decade.

However, we are still in a transitional phase and a great deal of uncertainty surrounds the path that will be followed in the coming years. The transition may be led by research interests (with researchers and research organisations, together with universities, assuming greater responsibility and a leading role in the dissemination and publication of their own results), or directed by the scientific publishing industry. This will impact, on the one hand, on the final configuration of the open access 
communication system and, on the other hand, on the role that may be reserved for university libraries within this system.

In the light of the experiences of the past ten years, particularly more recent developments in the open access policies from funding bodies, the aim of this paper is to reflect on the threats and opportunities involved in the transition to open access, in particular for university libraries.

\section{Introdução - O Acesso Aberto: origens, objetivos e formas}

Se o acesso aberto à literatura científica, tal como hoje o conhecemos, é um fenómeno com apenas uma dúzia de anos, a verdade é que as suas origens são mais remotas, e as suas raízes, nas tradições e nas práticas da comunicação académica e científica, são profundas.

A disseminação, circulação e partilha da informação e do conhecimento entre os pares (e as mentes curiosas) sempre foi o modo "normal" do inquérito intelectual, do funcionamento da atividade académica e científica, mesmo antes de ela se ter cristalizado e institucionalizado nas suas formas atuais. A ciência normal, tal como definida por Thomas Khun ${ }^{1}$, é cumulativa e baseada no acesso e utilização do conhecimento previamente construído. Já no século XII, Bernardo de Chartres afirmava: somos como anões aos ombros de gigantes, pois podemos ver mais coisas do que eles e mais distantes, não devido à acuidade da nossa vista ou à altura do nosso corpo, mas porque somos mantidos e elevados pela estatura de gigantes ${ }^{2}$.

Mas mesmo nos momentos das designadas revoluções científicas e de mudança de paradigma, como Newton reconheceu ${ }^{3}$, o acesso e a utilização do conhecimento anterior é a base para a construção do novo conhecimento. E nos dias de hoje, quando a ciência é chamada a ajudar

\footnotetext{
${ }^{1}$ KUHN, Thomas S. - La structure des révolutions scientifiques. [Paris]: Flammarion, cop. 1983. ISBN 2-08-081115-0.

2 Bernardo de Chartres, citado por SAliSBuRY, João de - Metalogicon, III, 4. In MCGARRY, Daniel D. - The Metalogicon of John of Salisbury: a twelfth-century defense of the verbal and logical arts of the Trivium. Los Angeles: University of California, 1971.

3 "What Des-Cartes [sic] did was a good step. You have added much several ways, \& especially in taking the colours of thin plates into philosophical consideration. If I have seen further it is by standing on the sholders [sic] of Giants." In NEWTON, Isaac - Carta para Robert Hooke. 15 de Fevereiro de 1676.
} 
a enfrentar e resolver ameaças e problemas globais, como os riscos de pandemias, uma das medidas para garantir que o contributo científico é mais rápido e eficiente é a disponibilização e partilha de toda a informação (desde os dados "em bruto" até às publicações) relevante sobre o problema ${ }^{4}$.

A distribuição alargada e a reutilização do conhecimento produzido pelo conjunto da comunidade parece ser, portanto, intrínseca à atividade científica, e uma condição necessária para garantir a geração de novo conhecimento de uma forma eficiente. A partilha de informação constitui também uma tradição enraizada na comunidade científica, desde o intenso intercâmbio epistolar que precedeu (e acompanhou) a criação das primeiras revistas científicas em meados do século XVII ${ }^{5}$, até à circulação de preprints $^{6}$ e reprints ${ }^{7}$ no século $\mathrm{XX}$, que esteve na origem do primeiro repositório científico moderno ${ }^{8}$. O acesso aberto retoma, continua e aprofunda esta velha tradição de partilha entre a comunidade científica.

A reunião ocorrida em Budapeste em dezembro de 2001, que juntou três dezenas de académicos, cientistas, bibliotecários e outras personalidades interessadas no sistema de comunicação da ciência, e de onde resultou a Declaração de Budapeste ${ }^{9}$ é geralmente considerada o momento fundador do atual movimento de acesso aberto. A Declaração de Budapeste foi o primeiro documento a estabelecer e definir o conceito e o termo "open access" (que em português se traduziu para acesso livre ou acesso

\footnotetext{
4 Tal como aconteceu com a gripe aviária. Ver BOGNER, Peter, [et. al.] - A global initiative on sharing avian flu data. [Em linha]. Nature. 442:7106 (31 august 2006), 981. [Consult a 1 de ago. de 2014]. Disponível na WWW em: <URL:http://www.nature.com/nature/journal/ v442/n7106/full/442981a.html>.

5 Le Journal des sçavans e The Philosophical Transactions of the Royal Society, ambas criadas em 1665.

6 Versão de artigo submetido para publicação numa revista científica, mas ainda não aceite.

7 Cópias de artigos publicados em revistas científicas.

8 O Arxiv, criado em 1991. CORNELL UNIVERSITY LIBRARY - arXiv.org. New York: CUL, 2010. [Consult. 1 ago. 2014]. Disponível na WWW em: <URL:http://arxiv.org>.

9 BUDAPEST OPEN ACCESS INNITIATIVE ORGANIZATION. Budapest Open Access Inniative. Budapest: BOAI, 2002. [Consult. 1 agos. 2014]. Disponível na WWW em: <http://www. budapestopenaccessinitiative.org/read>.
} 
aberto), e a apontar as duas formas ou vias para a sua concretização (as revistas e os repositórios de acesso aberto).

Tal como definido em Budapeste, o acesso aberto abrange toda a literatura (em primeiro lugar os artigos de revistas científicas com revisão por pares, mas também a outros tipos de publicações e documentos) que os investigadores produzem sem qualquer intenção de pagamento, e significa a disponibilização na Internet dessa literatura de forma gratuita e sem restrições de acesso.

A Budapest Open Access Initiative (BOAI), como também ficou conhecida esta iniciativa, resultou de uma crescente insatisfação com o sistema de comunicação científica, da consciência da necessidade e da possibilidade de resolver o problema da acessibilidade à literatura científica, bem como da maturação e convergência de diversas iniciativas e projetos no mesmo sentido, que já vinham a desenvolver-se previamente.

De facto, na origem do movimento do acesso aberto estão os problemas, limitações e contradições do sistema de comunicação da ciência, em particular os relacionados com as revistas científicas. $\mathrm{Na}$ segunda metade do século XX, o crescimento acentuado do volume da literatura científica foi acompanhado pela "comercialização" do sistema de comunicação da ciência, e pela perda do seu controlo por parte do mundo académico e científico. E a função essencial das revistas científicas - a divulgação de resultados de investigação para promover a disseminação do conhecimento e o avanço da ciência - foi obscurecida pelos objetivos comerciais das editoras (que no mesmo período viveram um processo de fusões e aquisições, que criou um mercado com contornos monopolistas).

Em resultado da evolução registada nas duas décadas anteriores, no final da década de 90 do século XX, no meio académico e entre os profissionais de informação, cresceu a consciência do agudizar da designada "crise dos periódicos" (aumento brutal do custo das assinaturas de revistas e consequentes cancelamentos de assinaturas por muitas bibliotecas), e das graves consequências que as limitações ao acesso à literatura produziam ao próprio sistema científico. Ao mesmo tempo, a generalização da utilização da Internet e da Web foi acompanhada por uma maior com- 
preensão das suas potencialidades e aplicações na publicação científica. A conjugação destes dois fenómenos resultou no aparecimento de diversas iniciativas que acabaram por convergir em Budapeste, dando origem ao atual movimento de acesso aberto.

A Declaração de Budapeste estabeleceu as duas formas ou vias para atingir o acesso aberto: publicação de revistas de acesso aberto (também designada de via dourada) e auto-arquivo/depósito em repositórios de acesso aberto (também designada via verde).

As revistas de acesso aberto não usam os direitos de autor (ou copyright) para restringir o acesso e o uso do material que publicam, não cobram assinatura nem taxas de acesso (à versão online) e recorrem a outras fontes (subsídios das instituições que as publicam, taxas de publicação ou Article Processing Charges - APC, pagamento da versão impressa, etc.) para cobrir as suas despesas.

A outra via para o acesso aberto é o auto-arquivo ou depósito, pelos autores ou seus representantes, dos artigos publicados nas revistas científicas (independentemente do seu modelo de publicação) em repositórios, disciplinares ou institucionais. Se o primeiro repositório disciplinar (o Arxiv) data de 1991, ou seja uma década antes da reunião de Budapeste, os repositórios institucionais têm uma história mais recente, relacionada com a BOAI. De facto, apesar de algumas iniciativas anteriores, foi a partir de finais de 2002 que se assistiu à proliferação de repositórios institucionais por parte de universidades e outros centros de investigação.

Devido ao seu carácter, os repositórios institucionais servem não apenas para armazenar e tornar acessível a literatura publicada em revistas científicas, mas igualmente os outros tipos de documentos produzidos no quadro das atividades de investigação e ensino (working papers, relatórios técnicos, comunicações a conferências, teses e dissertações, etc.). Por isso, para além da agenda do acesso aberto, os repositórios contribuem também para a promoção das instituições que os criam, aumentando a visibilidade, acesso e impacto dos resultados das suas atividades de investigação e ensino. 


\section{A evolução recente do Acesso Aberto em Portugal e no mundo}

$\mathrm{Na}$ última década, e em particular nos últimos seis anos, o acesso aberto conheceu um progresso notável, tanto em Portugal como no resto do mundo. Esse progresso pode ser avaliado através de diversos indicadores, como a evolução do número de repositórios e revistas de acesso aberto, do número e natureza das políticas de acesso aberto de instituições que realizam ou financiam investigação, ou do volume de publicações científicas e académicas disponíveis em acesso aberto e da percentagem que representam no universo da produção científica à escala global.

Em Portugal, as primeiras iniciativas de acesso aberto foram protagonizadas pela Universidade do Minho com a criação do RepositóriUM em 2003 e a definição de uma política institucional de auto-arquivo em $2004^{10}$. Nos anos seguintes registaram-se diversas iniciativas, como a criação do SciElo Portugal, para a publicação de revistas de acesso aberto, ou a criação de novos repositórios em diversas instituições de ensino superior. Mas foi o surgimento do projeto Repositório Científico de Acesso Aberto de Portugal (RCAAP), em 2008, que assinalou o início de uma nova dinâmica na evolução do acesso aberto em Portugal ${ }^{11}$.

O desenvolvimento, no âmbito do projeto RCAAP, de um conjunto de serviços (como o Portal RCAAP, o Serviço de Alojamento de Repositórios Institucionais, o Repositório Comum, o Serviço de Alojamento de Revistas Científicas, e outros) para as instituições e a comunidade académica, bem como de uma intensa atividade de comunicação e disseminação, impulsio-

10 RODRIGUES, Eloy; SARAIVA, Ricardo - RepositóriUM: 10 anos de acesso aberto ao conhecimento. [Em linha]. In Uma década de acesso aberto na UMinbo e no mundo. Braga: Universidade do Minho. Serviços de Documentação, 2013. ISBN 978-989-98704-0-6. p. 25-48. [Consult. 1 de ago. 2014]. Disponível na WWW em: <URL: http://hdl.handle.net/1822/27502>.

11 Para uma informação mais detalhada sobre a história e evolução do acesso aberto em Portugal ver: SARAIVA, Ricardo [et. al.] - Acesso Aberto à literatura científica em Portugal: o passado, o presente e o futuro. [Em linha]. Congresso Nacional de Bibliotecários, Arquivistas e Documentalistas "Integração, Acesso e Valor Social", 11. Lisboa: BAD, 2012. [Consult. 1 ago. 2014]. Disponível na WWW em: <URL: http://hdl.handle.net/1822/20542>. CARVALHO, José; MOREIRA, João M.; SARAIVA, Ricardo - O RCAAP e a evolução do acesso aberto em Portugal. In: Uma década de acesso aberto na UMinbo e no mundo [Em linha]. Braga: Universidade do Minho. Serviços de Documentação, 2013. ISBN 978-989-98704-0-6. p. 25-48. [Consult. 1 ago. 2014]. Disponível na WWW em: <URL: http://hdl.handle.net/1822/27502>. 
nou um rápido desenvolvimento do acesso aberto em Portugal, colocando o país numa posição destacada a nível internacional ${ }^{12}$. Neste período, quer o número de repositórios (que passou de 10 em 2008 para mais de 40 em 2014), quer o número de documentos portugueses disponíveis em acesso aberto no Portal RCAAP (que passou de pouco mais de 13000 no final de 2008 para mais de 150000 no início de 2014), cresceram a um ritmo ainda mais rápido do que o registado na generalidade dos países.

A evolução do número de revistas científicas e de repositórios de acesso aberto a nível mundial e nos últimos dozes anos foi, em qualquer caso, notável. Em relação às revistas de acesso aberto, passou-se de escassas centenas em 2002, para cerca de dez mil presentemente. O número global de revistas de acesso aberto esconde, no entanto, uma grande diversidade, quer quanto ao seu modelo de funcionamento (desde as revistas suportadas institucionalmente até às que cobram APCs, em alguns casos com valores elevados), quer quanto ao seu alcance, impacto e qualidade (de pequenas revistas eminentemente "locais", até revistas com elevado impacto e visibilidade). E, nos últimos anos, assistiu-se também à proliferação das chamadas "revistas predatórias"13, que oferecem a possibilidade de publicar qualquer artigo a troco do pagamento de uma taxa de publicação, revelando padrões de qualidade e de revisão por pares muito baixos, ou mesmo completamente inexistentes.

Quanto aos repositórios, que eram apenas algumas dezenas em 2002, viram aumentar o seu número até aos mais 2700 que atualmente existem. Quase todas as mais relevantes instituições de investigação do mundo (desde as universidades, aos laboratórios e centros de investigação),

12 OTTS, Claude - Up and away: open access in Portugal. [Em linha]. Educause. 2013. [Consult. 1 ago. 2014]. Disponível na WWW em: <URL:http://www.educause.edu/ero/article/ and-away-open-access-portugal>.

13 Os termos acesso aberto predatório e revistas predatórias começaram a ser usados por Jeffrey Beall para designar as revistas que enviam um grande número de convites para submeter artigos, cobram taxas de publicação e publicam artigos sem uma revisão editorial e controlo de qualidade adequada. Jeffrey Beall mantém uma lista de editores predatórios (Beall's List - http://scholarlyoa.com/publishers/), que apesar de ter sido contestada e criticada por diversas vezes, é considerada como uma fonte de informação útil por muitos investigadores e bibliotecários. Para mais informação consultar o artigo: Predatory openaccess publishing na Wikipedia. Wikipedia [Em linha]. [Consult. 1 agos. 2014]. Disponível na WWW em: <http://en.wikipedia.org/wiki/Predatory_open-access_publishing>. 
dispõem de repositórios institucionais para albergar a sua produção científica. Mas igualmente no domínio dos repositórios se verifica uma grande diversidade quer quanto à sua dimensão absoluta e relativa (a percentagem do output da instituição que conseguem albergar), quer quanto aos aspetos técnicos relacionados, entre outros, com a qualidade dos metadados e com a interoperabilidade com outros sistemas.

A área onde, nos últimos anos, o acesso aberto conheceu desenvolvimentos talvez ainda mais expressivos, e certamente com um impacto mais profundo e duradouro, foi a das políticas de acesso aberto por parte das instituições de investigação e das organizações financiadoras da ciência. As primeiras políticas de acesso aberto começaram a surgir no seio de instituições de investigação imediatamente após a Declaração de Budapeste: Southampton (Escola de Eletrónica e Informática) em 2002, CERN em 2003, Universidade do Minho e Queensland University of Technology em 2004. Mas foi a partir de 2006 que se começou a assistir à multiplicação das políticas (em muitos casos com carácter obrigatório, ou mandatório como são geralmente designadas) não apenas das instituições de investigação, mas crescentemente também dos financiadores, públicos (como o National Institute of Health - NIH- dos Estados Unidos em 2007) e privados (como o Wellcome Trust do Reino Unido em 2006), da investigação científica.

Presentemente existem mais de duzentas políticas institucionais de acesso aberto, e quase uma centena de políticas de financiadores, registadas no Registry of Open Access Repositories Mandatory Archiving Policies - ROARMAP ${ }^{14}$.

Nos últimos dois anos registaram-se inúmeros desenvolvimentos de grande importância no domínio das políticas de acesso aberto, em praticamente todas as regiões do mundo (desde a política do Australian

14 Ver UNIVERSITY OF SOUTHAMPTON. Electronics and Computer Science - Registry of Open Access Repositories Mandatory Archiving Policies (ROARMAP). Southampton: ECS, 2000-2012. [Consult. 1 ago. 2014]. Disponível na WWW em: <http://roarmap.eprints.org/>. 
Research Council ${ }^{15}$ até às leis sobre repositórios e acesso aberto do Perú ${ }^{16}$ e da Argentina ${ }^{17}$, ambas em 2013, ou já em 2014 a legislação mexicana de acesso aberto ${ }^{18}$, ou a política da academia de ciências da China ${ }^{19}$ ), mas pela sua relevância e impacto, quer no contexto português quer a nível global, iremos apenas deter-nos brevemente sobre alguns dos desenvolvimentos na Europa, incluindo a anunciada política de acesso aberto da Fundação para a Ciência e Tecnologia.

Em Julho de 2012 foram anunciadas novas iniciativas políticas pela Comissão Europeia, para o conjunto da União Europeia, e pelos Research Councils UK (RCUK), para o Reino Unido, que estão a marcar a evolução do acesso aberto, a nível mundial, desde então.

No Reino Unido, foi divulgado em julho de 2012 um relatório, conhecido por relatório Finch ${ }^{20}$ por ter sido produzido por uma comissão nomeada pelo ministro britânico da ciência e presidida pela dama Janet Finch. Este relatório expressou uma preferência, orientação e recomendação política a favor do acesso aberto dourado ("Gold OA" com o pagamento de taxas de publicações), desvalorizando e subalternizando o papel dos repositórios e o acesso aberto verde ("Green OA").

15 Ver mais informação em: AUSTRALIAN RESEARCH COUNCIL - ARC Open Access Policy (version 2013.1). [Em linha]. Consult. 1 ago. 2014]. Disponível na WWW em: <URL: http:// www.arc.gov.au/applicants/open_access.html>.

16 Ver PERU. CONSEJO NACIONAL DE CIENCIA, TECNOlogía E INNOVACión TECNOLÓGiCA Lei n. ${ }^{\circ}$ 3003. [Em linha]. [Consult. 1 ago. 2014]. Disponível na WWW em: <URL: http://portal. concytec.gob.pe/images/stories/images2013/portal/areas-institucion/dsic/ley-30035.pdf>.

17 Ver RePublica ARgEntina. Sistema NaCional De REPOSITORIOS Digitales - Recursos de Información. [Em linha]. [Consult. 1 ago. 2014]. Disponível na WWW em: <URL: http:// repositorios.mincyt.gob.ar/recursos.php $>$ http://repositorios.mincyt.gob.ar/recursos.php.

18 MEXICO. SECRETARÍA DE GOBERNACión - Diario Oficial de la Federación. [Em linha]. [Consult. 1 ago. 2014]. Disponível na WWW em:<URL:.http://www.dof.gob.mx/nota_detalle. php? codigo $=5345503 \&$ fecha $=20 \% 2$ F05\%2F2014>.

19 Ver CHINESE ACADEMY OF SCIENCES - Chinese Academy of Sciences policy statement on Open Access to articles from publicly funded scientific research projects. [Em linha]. [Consult. 1 ago. 2014]. Disponível na WWW em: <URL:. http://english.cas.cn/Ne/CASE/201405/ t20140516_121037.shtml>.

${ }^{20} \mathrm{FINCH}$, Janet Chair - Expanding access to published research: the Finch Report. Report of the UK Government Working Group on Expanding Access to Published Research. [Em linha]. London, 2012. [Consult. 1 jun. 2014]. Disponível na WWW em: <URL: http://www. researchinfonet.org/publish/finch>. 
Imediatamente após a publicação do relatório Finch, os RCUK anunciaram uma revisão da sua política de acesso aberto, no sentido de a alinharem com as recomendações daquele relatório. Em síntese, a política dos RCUK $^{21}$ passou de requerer o depósito das publicações em repositórios para requerer a publicação em revistas compatíveis com a sua política. As revistas compatíveis com a política dos RCUK são as que oferecem acesso aberto dourado (incluindo as revistas "híbridas"22), ou a possibilidade de depositar e disponibilizar os artigos em repositórios em acesso aberto, com um período de embargo que não pode ser superior a 6 meses, ou ambas as condições (caso em que os autores poderão escolher a opção que prefiram).

A orientação política Finch/RCUK afastou-se do que até aí tinha sido o ponto base das políticas de acesso aberto de entidades financiadoras: o requisito de depositar as publicações financiadas em repositórios, independentemente de essas políticas incluírem ou não apoio para a publicação em revistas de acesso aberto. E essa viragem política originou um intenso debate, tanto no meio académico do Reino Unido, como no movimento de acesso aberto a nível internacional.

Os críticos da política de acesso aberto Finch/RCUK têm vindo a reprovar essa política pelas suas implicações imediatas no Reino Unido, e também pelas consequências que está já a ter a nível global. Para o Reino Unido, a nova política implica no imediato o aumento da despesa total com publicações ${ }^{23}$ (pois é necessário manter a despesa com assinatura das revistas tradicionais, ao que se soma um montante crescente de taxas de publicação em revistas de acesso aberto ou híbridas), para

21 RESEARCH COUNCIL UK - Open Access. [Em linha]. [Consult 1 ago. 2014]. Disponível na WWW em: <URL: http://www.rcuk.ac.uk/research/outputs/>.

$22 \mathrm{O}$ acesso aberto dourado em revistas híbridas refere-se a revistas tradicionais, que cobram assinaturas ou outras taxas de acesso, e que simultaneamente disponibilizam a opção de acesso aberto no site do editor, através do pagamento de uma taxa de publicação. Para mais informação consultar o artigo Hybrid open access journals. [Em linha]. In WiKIPEDIA. [Consult. 1 ago. 2014], Disponível na WWW em: <URL: http://en.wikipedia. org/wiki/Hybrid_open_access_journal>.

23 BROOK, Michele - The sheer scale of hybrid journal publishing. In Open Access Working Group Blog. April 24, 2014. [Consult. 1 ago. 2014] Disponível na WWW em: <URL: http://access.okfn.org/2014/04/24/the-cost-of-academic-publishing >. 
além de desperdiçar o investimento que foi realizado na última década nos repositórios do Reino Unido.

A nível global, a política Finch/RCUK ofereceu à indústria de publicação científica o incentivo e a oportunidade de multiplicar as opções de acesso aberto híbrido, ao mesmo tempo que aumentam os períodos de embargo, pressionando os autores para que paguem as taxas de publicação, a fim de cumprir o definido nas políticas a que estão sujeitos. Este movimento de aumento do número de revistas híbridas e de aumento de período de embargo, foi claramente estimulado e potenciado pela política dos Research Councils UK, mas manifesta-se e tem consequências a nível mundial. Finalmente, ao não prever quaisquer limites ou medidas de controlo quanto ao valor das taxas de publicação, a nova política pode constituir também um incentivo ao aumento dos APCs, como poderá estar já a ocorrer ${ }^{24}$.

Esta orientação no Reino Unido contrasta com as políticas que ao mesmo tempo foram anunciadas pela Comissão Europeia. De facto, em julho de 2012, a Comissão divulgou três importantes documentos ${ }^{25}$ que estabelecem o acesso aberto como princípio geral no Espaço Europeu de Investigação, no âmbito do novo programa-quadro de investigação e inovação, Horizonte 2020 (H2020). E, contrariamente ao Reino Unido, a Comissão Europeia afirma explicitamente que tanto a "via verde" (repositórios) como a "via dourada" (revistas) são opções válidas para o acesso aberto, estabelecendo em qualquer caso o depósito de uma cópia dos artigos num repositório de acesso aberto como o requisito base da política para o $\mathrm{H} 2020$.

24 BJÖRK, Bo-Christer; SOLOMON, David - Developing an effective market for open access article processing charges. [Em linha]. London: Wellcome Trust, 2014. [Consult. a 1 ago. 2014] Disponível na WWW em: <URL:http://www.wellcome.ac.uk/stellent/groups/corporatesite/@ policy_communications/documents/web_document/wtp055910.pdf>.

25 EUROPEAN COMMISSION - Communication: a reinforced European Research area partnership for excellence and growth. [Em linha]. [Consult. 1 ago. 2014]. Disponível na WWW em: <URL:http://ec.europa.eu/research/science-society/document_library/pdf_06/ era-communication-partnership-excellence-growth_en.pdf $>$ e a COMISSÃO EUROPEIA Recomendação da Comissão de 17 de julbo de 2012 sobre o acesso à informação científica e a sua preservação. [Em linha]. [Consult. 1 ago. 2014]. Disponível na WWW em: <URL:http:// eur-lex.europa.eu/LexUriServ/LexUriServ.do?uri=OJ:L:2012:194:0039:0043:PT:PDF>. 
As orientações políticas anunciadas em 2012 começaram já a ser concretizadas e detalhadas nos documentos oficiais do $\mathrm{H} 2020^{26}$, confirmando-se que o acesso aberto à informação científica está definido como princípio geral. O novo modelo de contrato de subvenção estabelece uma obrigação geral de disseminação em acesso aberto dos resultados, indicando explicitamente que cada beneficiário deve depositar uma cópia de todas as publicações científicas, com revisão por pares que sejam respeitantes aos resultados do projeto, num repositório e assegurar o acesso aberto logo que possível e no limite até seis ou doze (para as ciências sociais e humanidades) meses após a publicação.

Esta orientação europeia foi também adotada em Portugal, na política de acesso aberto da Fundação para a Ciência e Tecnologia. Essa política, que tinha sido anunciada e divulgada numa versão preliminar para discussão pública em 2013, foi aprovada e publicada ${ }^{27}$ em maio de 2014 e engloba regras e recomendações para o acesso aberto a publicações sujeitas a revisão por pares e a dados resultantes de investigação científica financiada pela FCT, entrando em vigor a 5 de maio de 2014.

A política de acesso aberto a publicações científicas resultantes de investigação financiada pela FCT determina que as publicações que sejam sujeitas a revisão por pares ou outra forma de revisão científica, devem ser depositadas num dos repositórios da rede RCAAP logo que possível, de preferência por altura da aceitação da publicação. O acesso aberto ao texto integral das publicações deve ser permitido logo que possível, de preferência desde o momento da publicação ou depósito, mas admitem-se períodos de embargo variáveis de acordo com o tipo de publicação ou as áreas disciplinares. A política aplica-se a artigos em revistas cien-

26 Nomeadamente em EUROPEAN COMMISSION - Model grant agreement. [Em linha]. [Consult. 1 ago. 2014]. Disponível na WWW em: <URL:http://ec.europa.eu/research/ participants/portal/desktop/en/funding/reference_docs.html\#h2020-mga-gga> e em EUROPEAN COMMISSION - Guidelines on Open Access to scientific publications and research data in Horizon 2020. [Em linha]. [Consult. 1 ago. 2014]. Disponível na WWW em: <URL:http://eceuropa.eu/research/participants/data/ref/h2020/grants_manual/hi/oa_pilot/ h2020-hi-oa-pilot-guide_en.pdf $>$.

27 Ver PORTUgal. FundaÇão PARA A CiênCIA E A TeCNOlogia - e-Ciência: Política de Acesso Aberto. [Em linha]. [Consult. 1 ago. 2014]. Disponível na WWW em: <URL:https:// www.fct.pt/dsi/eciencia/index.phtml.pt>. 
tíficas, proceedings de conferências, posters, livros e capítulos de livros, monografias e teses de Mestrado e de Doutoramento.

\section{Considerações finais: por onde vamos e qual o papel das biblio- tecas universitárias nesse caminho?}

O crescimento do número de repositórios, de revistas e de políticas de acesso aberto, tem sido acompanhado também pelo aumento significativo da percentagem da produção científica a nível mundial que está disponível em acesso aberto. Ainda que tenham sido publicadas estimativas muito díspares nos últimos três anos ${ }^{28}$, os dados disponíveis apontam claramente para o crescimento do acesso aberto, que hoje já representará entre um terço e metade do volume total das publicações científicas dos últimos anos.

Esta crescente proporção dos artigos em acesso aberto e a pressão política em prol do acesso aberto, sobretudo das entidades financiadoras, indiciam que o acesso aberto já se tornou inevitável, e será o modo dominante na comunicação científica num futuro não muito distante. No entanto, se o destino final (predominância do acesso aberto) parece ser já conhecido, existem ainda muitas dúvidas e incertezas sobre os caminhos, os ritmos e as formas como ele será atingido. Essas dúvidas e

28 Entre outros, ver: GARGOURI, Yassine [et. al.] - Green and gold open access percentages and growth, by discipline. [Em linha]. In International Conference on Science and Technology Indicators (STI), 17 th. Montreal: OST, 2012. [Consult. 1 ago. 2014] Disponível na WWW em: URL<http://eprints.soton.ac.uk/340294>; ARCHAMBAULT, Eric [et. al.] - Proportion of open access peer-reviewed papers at the european and world levels - 2004-2011. Brussels: Science Metrix, 2013. [Consult. 1 ago. 2014] Disponível na WWW em: URL<http://www. science-metrix.com/pdf/SM_EC_OA_Availability_2004-2011.pdf>; BJÖRK, Bo-Christer [et. al.] - Anatomy of green open access. [Em linha]. Journal of the Association for Information Science and Technology. 65:2 (2014) .237-250. [Consult. 1 ago. 2014] Disponível na WWW em: URL:<http://www.openaccesspublishing.org/apc8/Personal\%20VersionGreenOa.pdf>; KHABSA, Madian; GILES, C. Lee - The number of scholarly documents on the web. [Em linha]. PLoS ONE. 9:5 (2014) e93949. [Consult. 1 ago. 2014]. Disponível na WWW em: <URL: http://www.plosone.org/article/info:doi/10.1371/journal.pone.0093949>; ARCHAMBAULT, Eric [et. al.] - Proportion of open access papers published in peer-reviewed journals at the european and world levels - 1996-2013. [Brussels]: Science Metrix, 2014. [Consult. 1 nov. 2014] Disponível na WWW em: URL<http://science-metrix.com/files/science-metrix/ publications/d_1.8_sm_ec_dg-rtd_proportion_oa_1996-2013_v11p.pdf >. 
incertezas estão relacionadas com uma questão central: quem, no sistema de comunicação científica, terá maior capacidade de liderar e conformar o processo de transição para o acesso aberto que já decorre e se prolongará por alguns anos?

A transição para o acesso aberto será liderada pela comunidade científica e as suas instituições (as universidades e outros centros de investigação e os financiadores da investigação), reassumindo o controlo do sistema de comunicação, como acontecia desde a criação das primeiras revistas até meados do século XX? Ou, pelo contrário, a transição será conduzida em função dos interesses e dos pontos de vista da indústria da informação científica e dos grandes grupos editoriais, mantendo o domínio estabelecido nas últimas décadas, que parecia ameaçado pelo acesso aberto?

No primeiro caso, as instituições de investigação e os financiadores terão de manter nos tempos mais próximos o foco no requisito básico de depósito de todas as publicações nos repositórios (ou seja, a denominada via verde). Este foco não impede que, simultaneamente, possa ser apoiada a publicação em acesso aberto (a designada via dourada), em especial através de iniciativas que não usem o pagamento de taxas de publicação. As políticas institucionais baseadas no depósito em repositórios são compatíveis (e em alguns casos podem ser até condição) com sólidas iniciativas editoriais institucionais ${ }^{29}$ para a publicação de livros e revistas de acesso aberto, bem como com iniciativas mais inovadoras ou "experimentais" como as revistas "overlay" 30.

Esta transição para o acesso aberto baseada nos repositórios, permitirá ainda desacoplar e individualizar os diferentes componentes, serviços e funções (registo, certificação, disseminação, arquivo, avaliação, etc.) que

29 Quer de editoras universitárias com experiências já muito consolidadas (como a Imprensa da Universidade de Coimbra, no caso português), quer novas iniciativas como a recentemente apresentada pela University College London, UCL Press (ver mais informação ver UNIVERSITY COLLEGE OF LONDON - UCL Library Services. UCL Press. [Em linha]. [Consult. 1 ago. 2014]. Disponível na WWW em: URL<http://www.ucl.ac.uk/library/ucl-press $>$.

30 Como o projeto a Episciences. Para mais informação ver CENTRE POUR LA COMMUNICATION SCIENTIFIQUE DIRECTE - épisciences.org. [Em linha]. [Consult. 1 ago. 2014]. Disponível na WWW em: URL:<http://episciences.org/>. 
hoje se encontram "empacotados" na publicação científica. Com os desenvolvimentos já registados, e com o potencial de inovação que existe em cada uma dessas áreas, é de crer que se registarão ganhos de qualidade e eficiência (incluindo redução de custos por unidade de publicação), através do uso de diferentes componentes e fornecedores de serviços, e da melhor forma de os combinar, em cada contexto.

Em alternativa a este cenário de transição, se a comunidade científica e as suas instituições não assumirem um maior controlo, o sistema de comunicação continuará a ser dirigido pela indústria da publicação científica. Esta, para além de não se ter revelado particularmente inovadora na última década, tem interesse em manter uma situação de controlo vertical da atividade (desde a publicação/registo - ou até antes, com ferramentas de pesquisa bibliográfica e gestão de referências - até aos sistemas e ferramentas de monitorização, métricas e avaliação da investigação), e terá neste cenário muito pouca pressão para a redução e transparência dos custos/preços. Aliás, como é natural, e parece ter sido demonstrado recentemente no Reino Unido relativamente a uma das principais editoras $^{31}$, a sua principal preocupação, que se reflete na política de preços, é o aumento (ou, no mínimo, a manutenção) das receitas que vêm obtendo num determinado mercado.

Neste cenário, a publicação de artigos em acesso aberto, sobretudo em revistas com taxas de publicação (incluindo um crescente número de revisas híbridas, como hoje acontece) poderá tornar-se no modo dominante mais rapidamente. Mas, pelo menos no período de transição durante o qual será necessário que as instituições continuem a subscrever as revistas "tradicionais" (incluindo as híbridas), isto terá custos acrescidos

31 De acordo com os dados recolhidos e divulgados por Tim Gowers os custos de assinatura das revistas da Elsevier pelas diferentes universidades inglesas não apresenta correlação com a população utilizadora ou o output científico, mas aparenta estar dependente da sua "fatura histórica" (as antigas despesas com assinaturas em papel) e da sua capacidade negocial. Sobre este assunto ver: GOWERS, Tim - Elsevier journals: some facts.[Em linha]. Gowers's Weblog. April 24, 2014. [Consult. 1 ago. 2014] Disponível na WwW em: URL:<http://gowers.wordpress.com/2014/04/24/elsevier-journals-some-facts/>; BROOK, Michele - The cost of academic publishing. [Em linha]. OKF Open Access Working Group Blog. March 24, 2014. [Consult. 1 ago. 2014]. Disponível na WWW em: URL:<http://access. okfn.org/2014/03/24/scale-hybrid-journals-publishing/>. 
para o sistema científico e as instituições académicas. E, mesmo após o período de transição, a manter-se o agrupamento das diversas funções/ serviços nas revistas científicas, poderá não ser criado o ambiente de competitividade e transparência que pressione no sentido da eficiência e do controlo dos custos e dos preços. Neste caso, poderá ocorrer o absurdo de as dificuldades de acesso à literatura, que hoje muitos investigadores ainda conhecem, serem substituídas pela dificuldade de esses mesmos investigadores publicarem os seus resultados.

As bibliotecas universitárias são, ou podem ser, importantes protagonistas neste processo de transição. E serão certamente profundamente influenciadas pela configuração final do acesso aberto que resulte deste período de transição. O papel que poderá ser desempenhado pelas bibliotecas no futuro sistema de comunicação científica dependerá da forma predominante do acesso aberto nos próximos anos.

No cenário de predomínio do acesso aberto dourado, com pagamento de taxas de publicação, a função das bibliotecas universitárias, e dos seus profissionais, poderá ficar limitada à gestão, ou apoio à gestão, do pagamento das taxas de publicação. Neste quadro, é provável que comecem a emergir modelos de venda/acordos de taxas de publicações em lote (os chamados big deals), tal como aconteceu nas últimas décadas com as assinaturas de revistas. E se hoje as vantagens dos big deals de assinatura, para as bibliotecas e as instituições e comunidades que servem, são crescentemente questionados, a sua substituição por big deals de publicação dificilmente poderá ser encarada como uma oportunidade para reforçar o papel das bibliotecas universitárias.

No cenário da transição liderada pela comunidade científica e suportada nos repositórios as bibliotecas universitárias poderão assumir um grande protagonismo. Em primeiro lugar, as universidades são um dos principais (e em muitos países, como Portugal, o principal) centros de investigação e de produção de literatura científica. E, na esmagadora maioria das universidades, os repositórios institucionais são geridos pelas bibliotecas (exclusivamente ou em conjunto com outros serviços).

Neste quadro, as bibliotecas universitárias poderão ter novas oportunidades para administrar e oferecer serviços e infraestruturas de gestão 
da informação científica (não apenas publicações, mas também dados científicos) produzida nas suas instituições. Para além das atividades e serviços que muitas já desenvolvem e oferecem (como a promoção do acesso aberto, a formação e suporte aos membros da instituição neste domínio, ou a manutenção e gestão de repositórios institucionais), as bibliotecas universitárias poderão ser chamadas a ter também um papel mais ativo na publicação e na gestão e curadoria dos dados científicos.

Como já referimos, uma das vias que tem vindo a ser crescentemente explorada, para criar um modelo sustentável para a publicação científica não baseado em taxas de publicação de valor elevado, é o desenvolvimento de iniciativas, plataformas e serviços institucionais para a publicação de revistas e livros em acesso aberto. Pela experiência e conhecimentos especializados que já reúnem, as bibliotecas universitárias são, em muitos casos, a unidade orgânica que na universidade estará em melhores condições para dirigir e desenvolver estas iniciativas.

No que diz respeito à gestão e curadoria dos dados científicos, ainda que essa seja uma área na qual muitos ainda não trabalham, existe também um grande potencial de intervenção para as bibliotecas universitárias e os seus profissionais. O desenvolvimento de competências e a criação de serviços no domínio do acesso e gestão dos dados científicos será um dos principais desafios e oportunidades para as bibliotecas universitárias na próxima década, como parece estar a ser compreendido por um crescente número de profissionais e organizações ${ }^{32}$.

O período de transição no sistema de comunicação e publicação científica que presentemente vivemos, como é natural nestes momentos, apresenta-se como turbulento, disruptivo e incerto, com vários riscos e múltiplas oportunidades, para os diversos atores envolvidos, desde

32 Diversas bibliotecas universitárias, e associações de bibliotecas universitárias, como a Ligue des Bibliothèques Européenes de Recherche - LIBER, têm vindo a desenvolver grupos de trabalho, estudos, recomendações e serviços sobre a gestão dos dados científicos. Uma boa síntese das possibilidades de intervenção das bibliotecas universitárias pode ser encontrada no documento da LIGUE DES BIBLIOTHÈQUES EUROPÉENES DE RECHERCHE (LIBER) - Ten recommendations for libraries to get started with research data management. [Em linha]. The Hague: LIBER, 2012. [Consult. 1 ago. 2014]. Disponível na WWW em: URL<http://libereurope.eu/blog/2012/08/24/ ten-recommendations-for-libraries-to-get-started-with-research-data-management $>$. 
os investigadores e académicos, autores da literatura científica, até aos editores e bibliotecas, tradicionalmente responsáveis pela disseminação e acesso a essa literatura.

Para as bibliotecas universitárias este período pode representar uma importante oportunidade para reafirmarem e reforçarem o seu papel no seio da instituição universitária. Neste tempo em que a intermediação "tradicional" (entre as fontes/recursos de informação "externos" e os utilizadores da organização - intermediação "de fora para dentro") está a perder importância, ameaçando a sua relevância, as bibliotecas têm a oportunidade de se reinventarem uma vez mais, afirmando-se num outro tipo de intermediação ("de dentro para fora"), oferecendo serviços que valorizem, disseminem e preservem o conhecimento produzido pelas comunidades universitárias em que estão inseridas.

Os próximos anos permitirão perceber se as bibliotecas universitárias saberão aproveitar esta oportunidade para que elas próprias, e as universidades que servem, assumam um maior papel, e sobretudo um maior controlo, do sistema de comunicação científica, ou se este continuará controlado por fornecedores externos de serviços de publicação, como aconteceu no último meio século.

\section{Referências bibliográficas}

ARCHAmbault, Eric [et. al.] - Proportion of open access papers published in peer-reviewed journals at the european and world levels: 1996-2013. [Em linha]. [Brussels]: Science Metrix, 2014. [Consult. 1 nov. 2014] Disponível na WWW em: <URL:http://science-metrix.com/files/science-metrix/publications/d_1.8_ sm_ec_dg-rtd_proportion_oa_1996-2013_v11p.pdf >.

ARCHAmBAult, Eric [et. al.] - Proportion of open access peer-reviewed papers at the european and world levels: 2004-2011. [Em linha]. Brussels: Science Metrix, 2013. [Consult. 1 ago. 2014] Disponível na WWW em: <URL:http://www.science-metrix.com/pdf/SM_EC_OA_Availability_2004-2011.pdf>.

Australian Research COUNCIL - ARC Open Access Policy (version 2013.1). [Em linha]. Consult. 1 ago. 2014]. Disponível na WWW em: <URL: http://Www.arc. gov.au/applicants/open_access.htm>. 
BIBLIOTECA NACIONAL DE PORTUGAL - Biblioteca nacional digital. Lisboa: BNP, 2002. [Consult. 21 Set. 2014]. Disponível na WWW em: <URL:http://purl.pt/ index/geral/PT/index.html>.

BJÖRK, Bo-Christer [et. al.] - Anatomy of green open access. [Em linha]. Journal of the Association for Information Science and Technology. 65:2 (2014) 237-250. [Consult. 1 ago. 2014] Disponível na WWW em: <URL:http://www.openaccesspublishing.org/apc8/Personal\%20VersionGreenOa.pdf $>$.

BJÖRK, Bo-Christer; SOLOMON, David - Developing an effective market for open access article processing charges. [Em linha]. London: Wellcome Trust, 2014. [Consult. 1 ago. 2014] Disponível na WWW em: <URL:http://www.wellcome. ac.uk/stellent/groups/corporatesite/@policy_communications/documents/ web_document/wtp055910.pdf>.

BOGNER, Peter, [et. al.] - A global initiative on sharing avian flu data. [Em linha]. Nature. 442:7106 (31 august 2006), 981. [Consult. 1 ago. 2014]. Disponível na WWW em: <URL:http://wWw.nature.com/nature/journal/v442/n7106/ full/442981a.html>.

BROOK, Michele - The cost of academic publishing. In Open Access Working Group Blog. March 24, 2014. [Consult 1 ago. 2014] Disponível na WWW em: <URL: http://access.okfn.org/2014/03/24/scale-hybrid-journals-publishing>.

BROOK, Michele - The sheer scale of hybrid journal publishing. In OKF Open Access Working Group Blog. April 24, 2014. [Consult. 1 ago. 2014] Disponível na WWW em: <URL: http://access.okfn.org/2014/04/24/the-cost-of-academic-publishing>.

BUDAPEST OPEN ACCESS INNITIATIVE ORgANIZATION. Budapest Open Access Inniative. Budapest: BOAI, 2002. [Consult. 1 ago. 2014]. Disponível na WWW em: <http://www.budapestopenaccessinitiative.org/read>.

CARVAlHO, José; MOREIRA, João M.; SARAIVA, Ricardo - O RCAAP e a evolução do acesso aberto em Portugal. In: Uma década de acesso aberto na UMinho e no mundo [Em linha]. Braga: Universidade do Minho. Serviços de Documentação, 2013. ISBN 978-989-98704-0-6. p. 25-48. [Consult. 1 ago. 2014] . Disponível na WWW em: <URL: http://hdl.handle.net/1822/27502>.

CENTRE POUR LA COMMUNICATION SCIENTIFIQUE DIRECTE - épisciences.org. [Em linha]. [Consult. 1 ago. 2014]. Disponível na WWW em: URL:<http://episciences.org/>. 
CHINESE ACADEMY OF SCIENCES - Chinese Academy of Sciences policy statement on Open Access to articles from publicly funded scientific research projects. [Em linha]. [Consult. 1 ago. 2014]. Disponível na WWW em: <URL:. http:// english.cas.cn/Ne/CASE/201405/t20140516_121037.shtml>.

COMISSÃo EUROPEIA - Recomendação da Comissão de 17 de julho de 2012 sobre o acesso à informação científica e a sua preservação. [Em linha]. [Consult. 1 ago. 2014]. Disponível na WWW em: <URL:http://eur-lex.europa.eu/LexUriServ/ LexUriServ.do?uri=OJ:L:2012:194:0039:0043:PT:PDF>.

CORNELL UNIVERSITY LIBRARY - arXiv.org. New York: CUL, 2010. [Consult. 1 ago. 2014]. Disponível na WWW em: <URL:http://arxiv.org>.

EUROPEAN COMMISSION - Communication: a reinforced European Research area partnership for excellence and growth [Em linha]. [Consult. 1 ago. 2014]. Disponível na WWW em: <URL:http://ec.europa.eu/research/science-society/document_ library/pdf_06/era-communication-partnership-excellence-growth_en.pdf>.

EUROPEAN COMMISSION - Guidelines on Open Access to scientific publications and research data in Horizon 2020. [Em linha]. [Consult. 1 ago. 2014]. Disponível na WWW em: <URL:http://eceuropa.eu/research/participants/data/ref/ h2020/grants_manual/hi/oa_pilot/h2020-hi-oa-pilot-guide_en.pdf>.

EUROPEAN COMMISSION - Model grant agreement. [Em linha]. [Consult. 1 ago. 2014]. Disponível na WWW em: <URL:http://ec.europa.eu/research/participants/ portal/desktop/en/funding/reference_docs.html\#h2020-mga-gga>.

FINCH, Janet Chair - Expanding access to published research: the Finch Report. Report of the UK Government Working Group on Expanding Access to Published Research. [Em linha]. London, 2012. [Consult. 1 jun. 2014]. Disponível na WWW em: <URL: http://www.researchinfonet.org/publish/finch>.

GARGOURI, Yassine [et. al.] - Green and gold open access percentages and growth, by discipline. [Em linha]. In International Conference on Science and Technology Indicators (STI), $17^{\text {th }}$. Montreal: OST, 2012. [Consult. 1 ago. 2014]. Disponível na WWW em: <URL: http://eprints.soton.ac.uk/340294/>.

GOWERS, Tim - Elsevier journals: some facts. In Gowers's Weblog. April 24, 2014. [Consult. 1 ago. 2014] Disponível na WWW em: <URL: http://gowers.wordpress. com/2014/04/24/elsevier-journals-some-facts/>. 
Hybrid open access journals. [Em linha]. In WIKIPEDIA. [Consult. 1 ago. 2014]. Disponível na WWW em: <URL: http://en.wikipedia.org/wiki/ Hybrid_open_access_journal>.

KHABSA, Madian; GILES, C. Lee - The number of scholarly documents on the web. [Em linha]. PLoS ONE. 9:5 (2014) e93949. [Consult. 1 ago. 2014]. Disponível na WWW em: <URL: http://www.plosone.org/article/info:doi/10.1371/journal. pone.0093949>.

KUHN, Thomas S. - La structure des révolutions scientifiques. [Paris]: Flammarion, cop. 1983. ISBN 2-08-081115-0.

LIGUE DES BIBLIOTHÈQues EUROPÉENES DE RECHERCHE (LIBER) - Ten recommendations for libraries to get started with research data management. [Em linha]. The Hague: LIBER, 2012. [Consult. 1 ago. 2014]. Disponível na WWW em: URL<http://libereurope.eu/news/ten-recommendations-for-libraries-toget-started-with-research-data-management>.

MEXICO. SECRETARía DE GOBERNACIÓN - Diario Oficial de la Federación. [Em linha]. [Consult. 1 ago. 2014]. Disponível na WWW em: <URL:.http://www.dof. gob.mx/nota_detalle.php?codigo=5345503\&fecha=20\%2F05\%2F2014>.

PERU. CONSEJO NACIONAL DE Ciencia, TeCnOlogía E InNOVACión TeCnOlóGICA - Lei n. ${ }^{\circ}$ 3003. [Em linha]. [Consult. 1 ago. 2014]. Disponível na WWW em: <URL: http://portal.concytec.gob.pe/images/stories/images2013/portal/ areas-institucion/dsic/ley-30035.pdf $>$.

Portugal. FundaÇÃo PARA A CiênCIA E A TECNOlogia - e-Ciência: Política de Acesso Aberto. [Em linha]. [Consult. 1 ago. 2014]. Disponível na WWw em: <URL:https://www.fct.pt/dsi/eciencia/index.phtml.pt>.

POTTS, Claude - Up and away: open access in Portugal. [Em linha]. Educause. 2013. [Consult. 1 ago. 2014]. Disponível na WWW em: <URL:http://www.educause.edu/ero/article/and-away-open-access-portugal $>$.

Predatory open-access publishing na Wikipedia. Wikipedia [Em linha]. [Consult. 1 ago. 2014]. Disponível na WWW em: <http://en.wikipedia.org/wiki/ Predatory_open-access_publishing $>$.

REPUBliCA ARGENTINA. SISTEMA NACIONAL DE REPOSITORIOS DigitAlES - Recursos de Información. [Em linha]. [Consult. 1 ago. 2014]. Disponível na WWW em: <URL: http://repositorios.mincyt.gob.ar/recursos.php>. 
RESEARCH COUNCIL UK - Open Access. [Em linha]. [Consult. 1 ago. de 2014]. Disponível na WWW em: <URL: http://www.rcuk.ac.uk/research/outputs/>. RODRIGUES, Eloy; SARAIVA, Ricardo - RepositóriUM: 10 anos de acesso aberto ao conhecimento. [Em linha]. In Uma década de acesso aberto na UMinho e no mundo. Braga: Universidade do Minho. Serviços de Documentação, 2013. ISBN 978-989-98704-0-6. p. 25-48. [Consult. 1 ago. 2014]. Disponível na WWW em: <URL: http://hdl.handle.net/1822/27502>.

SALISBURY, João de - Metalogicon, III, 4. In MCGARRY, Daniel D. - The Metalogicon of John of Salisbury: a twelfth-century defense of the verbal and logical arts of the Trivium. Los Angeles: University of California, 1971.

SARAIVA, Ricardo [et. al.] - Acesso Aberto à literatura científica em Portugal: o passado, o presente e o futuro. [Em linha]. Congresso Nacional de Bibliotecários, Arquivistas e Documentalistas “Integração, Acesso e Valor Social”, 11. Lisboa: BAD, 2012. [Consult. 1 ago. 2014]. Disponível na WWW em: <URL: http://hdl.handle.net/1822/20542>.

UNIVERSITY COLLEGE OF LONDON - UCL Library Services. UCL Press. [Em linha]. [Consult. 1 ago. 2014]. Disponível na WWW em: URL<http://Www.ucl.ac.uk/ library/ucl-press $>$.

UNIVERSITY OF SOUTHAMPTON. Electronics and Computer Science - Registry of Open Access Repositories Mandatory Archiving Policies (ROARMAP). Southampton: ECS, 2000-2012. [Consult. 1 ago. 2014]. Disponível na WWW em: $<$ http://roarmap.eprints.org/>. 
José Augusto Cardoso Bernardes é Professor da Faculdade de Letras da Universidade de Coimbra e Diretor da Biblioteca Geral da Universidade

Ana Maria Eva Miguéis é coordenadora do Serviço Integrado das Bibliotecas da Universidade de Coimbra

Carla Ferreira é bibliotecária nos Serviços de Biblioteca e Documentação da Faculdade de Letras da Universidade de Coimbra. 


\section{Série Documentos}

Imprensa da Universidade de Coimbra

Coimbra University Press

2015

C •

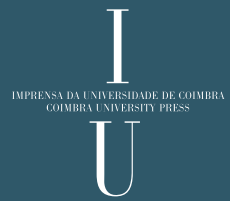

